\title{
Cochrane
México
}

\section{Drenaje peritoneal versus laparotomía como abordaje para enterocolitis necrosante perforada en recién nacidos pretérmino de bajo peso}

\author{
Paula Loyola-Nieto ${ }^{1}$, Horacio Márquez-González², Leticia A. Barajas-Nava ${ }^{3 *}$ y Jaime Nieto-Zermeño ${ }^{4}$ \\ ${ }^{1}$ Facultad de Medicina, Universidad Panamericana; ${ }^{2}$ Departamento de Investigación Clínica, Hospital Infantil de México Federico Gómez; ${ }^{3}$ Unidad \\ de Investigación de Medicina Basada en Evidencias, Centro Asociado Cochrane, Hospital Infantil de México Federico Gómez; ${ }^{4}$ Dirección General, \\ Hospital Infantil de México Federico Gómez. Ciudad de México, México
}

\begin{abstract}
Resumen
El desarrollo de enterocolitis necrosante, con la consecuente perforación intestinal, es frecuente en los recién nacidos pretérmino. El tratamiento estándar de la perforación intestinal es quirúrgico. Sin embargo, se sugiere que la inserción de un drenaje en el abdomen puede ser efectivo para tratar esta afección. Se resumen los resultados de una revisión sistemática Cochrane que compara la efectividad del drenaje peritoneal con la de la laparotomía en neonatos con enterocolitis necrosante perforada.
\end{abstract}

Palabras clave: Recién nacido. Pretérmino. Enterocolitis necrosante. Drenaje peritoneal. Laparotomía. Revisión sistemática.

\section{Peritoneal drainage versus laparotomy for perforated necrotizing enterocolitis in preterm low birth weight infants}

\begin{abstract}
Necrotizing enterocolitis is common in preterm newborns, with consequent intestinal perforation. The standard treatment for intestinal perforation is surgery. However, it is suggested that inserting a drain into the abdomen may be effective in treating this condition. This document summarizes the results of a Cochrane systematic review comparing the effectiveness of peritoneal drainage with laparotomy in neonates with perforated necrotizing enterocolitis.
\end{abstract}

Keywords: Newborns. Preterm. Necrotizing enterocolitis. Peritoneal drainage. Laparotomy. Systematic review.

\section{Correspondencia:}

*Leticia A. Barajas-Nava

E-mail: Leticia.barajas@cochrane.es
Fecha de recepción: 05-11-2020

Fecha de aceptación: 20-11-2020

DOI: 10.24875/BMHIM.20000358
Disponible en internet: 23-07-2021

Bol Med Hosp Infant Mex. 2021;78(4):331-334

www.bmhim.com

1665-1146/C 2020 Hospital Infantil de México Federico Gómez. Publicado por Permanyer. Este es un artículo open access bajo la licencia CC BY-NC-ND (http://creativecommons.org/licenses/by-nc-nd/4.0/). 


\section{Introducción}

Una revisión sistemática Cochrane evaluó la eficacia y la seguridad de la terapia con drenaje peritoneal en comparación con la laparotomía para el tratamiento de la enterocolitis necrosante o de la perforación intestinal espontánea en prematuros con bajo peso al nacer'. Este documento resume los hallazgos obtenidos en la revisión.

Desde que se crearon las unidades de cuidados intensivos neonatales, y a partir de la era del surfactante $^{2}$, es cada vez más frecuente que los pacientes prematuros sobrevivan. A escala mundial, se estima que aproximadamente 15 millones de recién nacidos son pretérmino, lo cual representa una tasa del $11 \%$ de los nacimientos en todo el mundo ${ }^{3}$. Sin embargo, la presentación de comorbilidad es frecuente, y entre ella, la enterocolitis necrosante presenta una incidencia del $3-16.8 \%{ }^{4}$.

La enterocolitis necrosante es una afección compleja y multifactorial que se desencadena, principalmente, por la suma de los siguientes fenómenos: secuestro sanguíneo intestinal secundario a hipoxia, subdesarrollo del sistema inmunitario del tracto gastrointestinal (aumenta la susceptibilidad) y disbiosis (alteraciones en la flora intestinal y crecimiento anormal de bacterias patógenas) $)^{5,6}$. La clasificación de Bell modificada de la enterocolitis necrosante incluye criterios clínicos y radiológicos para establecer sus estadios ${ }^{7}$. Con la detección oportuna, se espera que al menos la tercera parte de los casos permanezcan en un estadio de sospecha; sin embargo, del $25-40 \%$ progresarán a los estadios avanzados, de los cuales el desenlace más grave es la muerte por coagulación intravascular diseminada (Tabla 1).

Existe otra variedad de presentación, que es la perforación intestinal espontánea, también conocida como perforación aislada o perforación intestinal focal del recién nacido. Se caracteriza por ser una perforación única que en general se encuentra en el íleon terminal y se presenta en neonatos prematuros con muy bajo peso al nacer $(<1500 \mathrm{~g})$ y peso sumamente bajo al nacer $(<1000 \mathrm{~g})$. Cabe destacar que la perforación intestinal espontánea es una condición clínica separada de la enterocolitis necrosante ${ }^{8}$.

El tratamiento en las fases I y II se basa principalmente en medidas de soporte, como ayuno, soluciones intravenosas (o nutrición parenteral), antimicrobianos y, en caso de inestabilidad hemodinámica, ventilación mecánica asistida, inotrópicos y vasopresores. Cuando existen datos de isquemia intestinal o perforación, el abordaje debe ser invasivo:
Tabla 1. Clasificación de Bell modificada de la enterocolitis necrosante

\begin{tabular}{|c|c|}
\hline Estadio & Signos y síntomas \\
\hline Estadio I (sospecha) & $\begin{array}{l}\text { Irritabilidad, letargia, temperatura } \\
\text { inestable, aumento de residuos } \\
\text { gástricos, distensión abdominal y } \\
\text { emesis }\end{array}$ \\
\hline Estadio IA & $\begin{array}{l}\text { Signos y síntomas descritos sin } \\
\text { sangrado en heces }\end{array}$ \\
\hline Estadio IB & $\begin{array}{l}\text { Signos y síntomas descritos con } \\
\text { sangrado oculto en heces }\end{array}$ \\
\hline Estadio II (diagnóstico) & $\begin{array}{l}\text { Signos y síntomas descritos } \\
\text { anteriormente más celulitis de la } \\
\text { pared abdominal o plastrón en el } \\
\text { cuadrante inferior derecho }\end{array}$ \\
\hline Estadio IIA & Enfermedad moderada \\
\hline Estadio IIB & $\begin{array}{l}\text { Enfermedad moderada más acidosis } \\
\text { metabólica y trombocitopenia } \\
\text { moderada }\end{array}$ \\
\hline Estadio III (avanzada) & $\begin{array}{l}\text { Datos francos de inestabilidad } \\
\text { hemodinámica y abdomen agudo con } \\
\text { evidencia radiográfica de perforación. } \\
\text { Es la forma más grave y los pacientes } \\
\text { se encuentran en un estadio crítico } \\
\text { con hipotensión, bradicardia, apnea } \\
\text { grave, peritonitis, acidosis metabólica } \\
\text { y respiratoria, neutropenia y } \\
\text { coagulación intravascular diseminada }\end{array}$ \\
\hline Estadio IIIA & Los intestinos permanecen intactos \\
\hline Estadio IIIB & $\begin{array}{l}\text { Se presenta perforación intestinal } \\
\text { que se manifiesta como } \\
\text { neumoperitoneo }\end{array}$ \\
\hline
\end{tabular}

- El drenaje peritoneal primario, cuyo objetivo es paliativo, disminuye la presión intrabdominal y produce una mejora secundaria en la circulación intestinal mediante la punción y la liberación de líquido de ascitis y gas.

- La laparotomía suele implicar la resección del segmento intestinal afectado y su anastomosis o la formación de un estoma que permita la evacuación (con posterior reanastomosis) cuando la lesión ha sido extensa.

Durante los procedimientos quirúrgicos existe un alto riesgo de muerte de los recién nacidos pretérmino y con bajo peso debido a la inestabilidad hemodinámica y a la posibilidad de complicaciones y secuelas irreversibles, por lo que representan un reto para los médicos que diariamente enfrentan estos escenarios. Por ello, el objetivo de esta revisión fue evaluar los beneficios y los riesgos del drenaje peritoneal en comparación con la laparotomía como tratamiento quirúrgico inicial para 
la enterocolitis necrosante perforada o la perforación intestinal espontánea en recién nacidos prematuros.

\section{Métodos}

La revisión ${ }^{1}$ que se describe se realizó de acuerdo con la metodología Cochrane para revisiones sistemáticas de intervenciones ${ }^{9}$. Se incluyeron ensayos clínicos controlados aleatorizados y quasi-aleatorizados en los que se evaluó el drenaje peritoneal o la laparotomía como tratamiento quirúrgico inicial en recién nacidos prematuros (< 37 semanas de gestación) o de bajo peso al nacer $(<2500 \mathrm{~g})$ con enterocolitis necrosante perforada o perforación intestinal espontánea.

El resultado principal evaluado en la revisión fue la mortalidad a los 28 y 90 días, 6 meses y 1 año. También se evaluaron las siguientes variables: necesidad de nutrición parenteral por más de 3 meses después de la cirugía inicial; enterocolitis necrosante recurrente; necesidad de laparotomía en cualquier momento después del procedimiento primario y al día 7 ; desarrollo de absceso abdominal, fístula abdominal entero-entérica o entero-cutánea, estenosis intestinal o fístula vesical; días de inicio de nutrición enteral; tiempo de estancia intrahospitalaria; neurodesarrollo; y coste por cada paciente que sobrevive hasta el alta.

Se realizaron búsquedas de la literatura científica en las bases de datos de Cochrane Central Register of Controlled Trials (CENTRAL), The Cochrane Library 2010 (Issue 3), MEDLINE (1966 a julio 2010), EMBASE (1980 a julio 2010) y CINAHL (1982 a julio 2010), y en las sociedades académicas de pediatría, sin restricción de idioma. También se realizaron búsquedas en los registros de ensayos clínicos (Clinicaltrials.gov, Controller-trials.com y who.int/ictrp). Para el proceso de búsqueda se utilizaron términos MeSH y palabras clave (infant, newborn, low birth weight, premature, necrotizing enterocolitis, perforated NEC, peritoneal drainage, laparotomy).

El proceso de identificación, selección de estudios, extracción y manejo de datos fue realizado por dos revisores independientes. Los desacuerdos se resolvieron mediante discusión y con la intervención de un tercer revisor. La calidad metodológica de los estudios incluidos se evaluó con la herramienta de riesgo de sesgo ${ }^{9}$. Los datos se analizaron de acuerdo con los estándares del Cochrane Neonatal Review Group.

El análisis de datos se realizó mediante síntesis cuantitativa: para los resultados dicotómicos se utilizaron el riesgo relativo (RR) y la diferencia de riesgos; para los datos continuos, la diferencia de medias; y para todos los resultados, los intervalos de confianza del 95\% (IC95\%). Se evaluó la heterogeneidad estadística mediante un valor estándar de $I^{2}$. Para el análisis se utilizó el software estadístico de la Colaboración Cochrane, Review Manager (RevMan) Versión 5.3, utilizando el modelo de efectos fijos ${ }^{10}$.

Se realizó análisis de subgrupos para peso al nacer $\leq 1000 \mathrm{~g}$ y $>1000 \mathrm{~g}$.

\section{Resultados}

Mediante la búsqueda en la literatura se identificaron 22 estudios potencialmente elegibles. Se excluyeron 20 porque no eran estudios aleatorizados, con lo cual únicamente dos cumplieron los criterios de elegibilidad para su inclusión.

En un ensayo clínico ${ }^{11}$ controlado aleatorizado multicéntrico (15 centros en los Estados Unidos y Canadá) que incluyó 117 recién nacidos prematuros ( $\leq 34$ semanas de gestación, peso al nacer $<1500 \mathrm{~g}$ ) con evidencia de enterocolitis necrosante perforada o perforación intestinal espontánea, se evaluó el drenaje peritoneal primario en comparación con la laparotomía primaria. Las variables evaluadas fueron la mortalidad a 90 días después de la intervención, la dependencia de la nutrición parenteral posoperatoria y la duración de la estancia hospitalaria. En un segundo ensayo clínico $^{12}$ controlado aleatorizado multicéntrico (31 unidades quirúrgicas neonatales) se incluyeron 69 neonatos prematuros de peso extremadamente bajo al nacer $(<1000 \mathrm{~g})$ con evidencia de perforación intestinal. En este estudio se evaluaron la supervivencia al mes y a los 6 meses de la intervención, la duración de la estancia hospitalaria, la dependencia de la ventilación y de la nutrición parenteral, y el tiempo hasta la alimentación enteral completa.

De acuerdo con los resultados del metaanálisis que combinó los dos estudios ${ }^{1}$, no se encontraron diferencias estadísticamente significativas en la mortalidad en los 28 días posteriores al procedimiento primario entre el drenaje peritoneal y la laparotomía (RR: 0.99; IC95\%: 0.641.52). En cuanto a la mortalidad en los neonatos de peso al nacer < $1000 \mathrm{~g}$, el análisis no mostró diferencias estadísticamente significativas entre el drenaje peritoneal y la laparotomía (RR: 1.15; IC95\%: 0.72-1.88).

Para la mortalidad dentro de los 90 días posteriores al procedimiento primario tampoco se observaron diferencias estadísticamente significativas en el análisis combinado de los datos (RR: 1.05; IC95\%: 0.7-1.55). De igual forma, en los neonatos de peso al nacer < 1000 g, el metaanálisis no mostró diferencias estadísticamente significativas entre el drenaje peritoneal y la laparotomía (RR: 1.12; IC95\%: 0.74-1.72). Sin embargo, 
el análisis combinado de los resultados demostró un aumentó estadísticamente significativo en el riesgo de necesidad de laparotomía en el grupo de drenaje peritoneal primario dentro de los primeros 7 días (RR: 6.37; IC95\%: 1.88-21.5) y en cualquier momento después del procedimiento primario (RR: 0.49; IC95\%: 0.39-0.61).

Para el resto de los resultados evaluados, como la necesidad de nutrición parenteral total, la duración de la estancia hospitalaria, la enterocolitis necrotizante recurrente o cualquier otra complicación (absceso abdominal, fístula intestinal, fístula de vejiga urinaria), no se encontraron diferencias estadísticamente significativas entre el drenaje peritoneal y la laparotomía.

\section{Conclusiones}

La evidencia no indica diferencias significativas de beneficio $o$ daño entre el drenaje peritoneal y la laparotomía. Es posible que el tamaño de muestra tan reducido de los estudios no permita distinguir algunas diferencias clínicamente relevantes. Se requieren nuevos ensayos clínicos de gran tamaño, con seguimiento a largo plazo y que consideren la estratificación de acuerdo con el diagnóstico preoperatorio y el coste-efectividad del drenaje peritoneal sobre la laparotomía.

Con la información existente, no es posible establecer recomendaciones respecto a la elección del tratamiento quirúrgico inicial para la enterocolitis necrotizante perforada o la perforación intestinal espontánea en los recién nacidos prematuros.

\section{Implicaciones clínicas}

El propósito de la revisión analizada fue comparar dos procedimientos quirúrgicos que son complementarios entre sí. El uso de drenajes en la enterocolitis necrosante se remonta a la década de 1970, con fines de paliar las complicaciones intestinales en los neonatos de muy bajo peso con inestabilidad hemodinámica; en algunos casos se evitó la necesidad de cirugía. No obstante, empíricamente, algunos grupos emplean los drenajes como tratamiento único. De acuerdo con la evidencia, el metaanálisis mostró que los neonatos tratados con drenaje peritoneal finalmente requerirán cirugía. Así mismo, la revisión reportó que la mortalidad comparada entre ambos procedimientos no es diferente en los neonatos con peso < $1000 \mathrm{~g}$. Esto probablemente se explica por la heterogeneidad de la población y de los centros hospitalarios sede de los estudios incluidos.

Tomando en cuenta los resultados reportados, es posible justificar el uso de drenajes como una alternativa paliativa para ganar tiempo y poder mejorar las condiciones clínicas de los pacientes, hasta que cuenten con las condiciones de estabilidad hemodinámica requeridas para una intervención quirúrgica como la laparotomía.

Considerar la evidencia científica para la toma de decisiones clínicas permite garantizar mejores resultados y disminuye las posibles complicaciones.

\section{Responsabilidades éticas}

Protección de personas y animales. Los autores declaran que para esta investigación no se han realizado experimentos en seres humanos ni en animales.

Confidencialidad de los datos. Los autores declaran que en este artículo no aparecen datos de pacientes.

Derecho a la privacidad y consentimiento informado. Los autores declaran que en este artículo no aparecen datos de pacientes.

\section{Conflicto de intereses}

Los autores declaran que no existe ningún conflicto de intereses.

\section{Financiamiento}

Ninguno.

\section{Bibliografía}

1. Rao SC, Basani L, Simmer K, Samnakay N, Deshpande G. Peritoneal drainage versus laparotomy as initial surgical treatment for perforated necrotizing enterocolitis or spontaneous intestinal perforation in preterm low birth weight infants. Cochrane Database Syst Rev. 2011;(15):CD006182.

2. Sardesai S, Biniwale M, Wertheimer F, Garingo A, Ramanathan R. Evolution of surfactant therapy for respiratory distress syndrome: past, present, and future. Pediatr Res. 2017;81:240-8.

3. Walani SR. Global burden of preterm birth. Int J Gynaecol Obstet. 2020:150:31-3.

4. Alsaied A, Islam N, Thalib L. Global incidence of necrotizing enterocolitis: a systematic review and meta-analysis. BMC Pediatr. 2020;20:344.

5. Bazacliu C, Neu J. Necrotizing enterocolitis: long term complications. Curr Pediatr Rev. 2019;15:115-24

6. Eaton S, Rees CM, Hall NJ. Current research on the epidemiology, pathogenesis, and management of necrotizing enterocolitis. Neonatology. 2017; $111: 423-30$

7. De Bernardo G. Necrotizing enterocolitis: from diagnosis to therapy. Curr Pediatr Rev. 2019;15:67.

8. Frost BL, Modi BP, Jaksic T, Caplan MS. New medical and surgical insights into neonatal necrotizing enterocolitis: a review. JAMA Pediatr. 2017;171:83-8.

9. Higgins JPT, Green S, editores. Cochrane Handbook for Systematic Reviews of Interventions Version 5.1.0 [updated March 2011]. The Cochrane Collaboration; 2011. Disponible en: www.cochrane-handbook.org.

10. The Nordic Cochrane Centre, The Cochrane Collaboration. Review Manager (RevMan). Version 5.3. Copenhagen: The Nordic Cochrane Centre, The Cochrane Collaboration; 2014.

11. Moss RL, Dimmitt RA, Barnhart DC, Sylvester KG, Brown RL, Powell DM, et al. Laparotomy versus peritoneal drainage for necrotizing enterocolitis and perforation. N Engl J Med 2006;354:2225-34.

12. Rees CM, Eaton S, Kiely EM, Wade AM, McHugh K, Pierro A. Peritoneal drainage or laparotomy for neonatal bowel perforation? A randomized controlled trial. Annals of Surgery. 2008;248:44-51. 